\title{
Ernst, Julia
}

\section{Aktuelle Herausforderungen bei der Schulverpflegung an Gemeinschaftsschulen}

Haushalt in Bildung \& Forschung 6 (2017) 1, S. 47-61

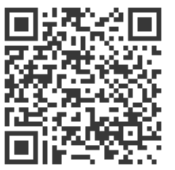

Quellenangabe/ Reference:

Ernst, Julia: Aktuelle Herausforderungen bei der Schulverpflegung an Gemeinschaftsschulen - In:

Haushalt in Bildung \& Forschung 6 (2017) 1, S. 47-61 - URN: urn:nbn:de:0111-pedocs-191319 - DOI: $10.25656 / 01: 19131$

https://nbn-resolving.org/urn:nbn:de:0111-pedocs-191319

https://doi.org/10.25656/01:19131

in Kooperation mit / in cooperation with:

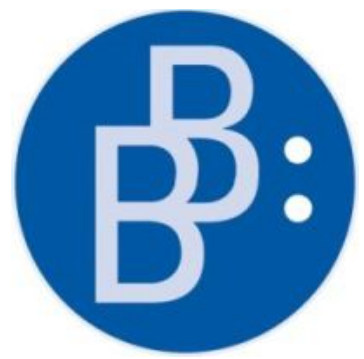

https://www.budrich.de

\section{Nutzungsbedingungen}

Gewährt wird ein nicht exklusives, nicht übertragbares, persönliches und beschränktes Recht auf Nutzung dieses Dokuments. Dieses Dokument ist ausschließlich für den persönlichen, nicht-kommerziellen Gebrauch bestimmt. Die Nutzung stellt keine Übertragung des Eigentumsrechts an diesem Dokument dar und gilt vorbehaltlich der folgenden Einschränkungen: Auf sämtlichen Kopien dieses Dokuments müssen alle Urheberrechtshinweise und sonstigen Hinweise auf gesetzlichen Schutz beibehalten werden. Sie dürfen dieses Dokument nicht in irgendeiner Weise abändern, noch dürfen Sie dieses Dokument für öffentliche oder kommerzielle Zwecke vervielfältigen, öffentlich ausstellen, aufführen, vertreiben oder anderweitig nutzen.

Mit der Verwendung dieses Dokuments erkennen Sie die Nutzungsbedingungen an.

\section{Terms of use}

We grant a non-exclusive, non-transferable, individual and limited right to using this document.

This document is solely intended for your personal, non-commercial use. Use of this document does not include any transfer of property rights and it is conditional to the following limitations: All of the copies of this documents mus retain all copyright information and other information regarding legal protection. You are not allowed to alter this document in any way, to copy it for public or commercial purposes, to exhibit the document in public, to perform, distribute or otherwise use the document in public.

By using this particular document, you accept the above-stated conditions of use.

\section{Kontakt / Contact:}

\section{peDOCS}

DIPF | Leibniz-Institut für Bildungsforschung und Bildungsinformation Informationszentrum (IZ) Bildung

E-Mail: pedocs@dipf.de

Internet: www.pedocs.de

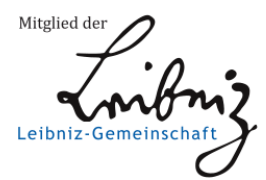


6. Jahrgang Heft 1

2017

峁

仓

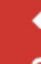

()

음

닌

亲

c

ชิ

은

\)

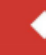

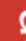

8

垔

ह

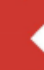

$+$

$\underline{\xi}$

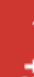

$\pm$

$\frac{9}{7}$

气

$\mathbb{1}$

চ

ISSN 2193-8806

\section{Haushalt in \\ Forschung}

\section{Qualifizierungsarbeiten Konsum - Ernährung - Gesundheit}
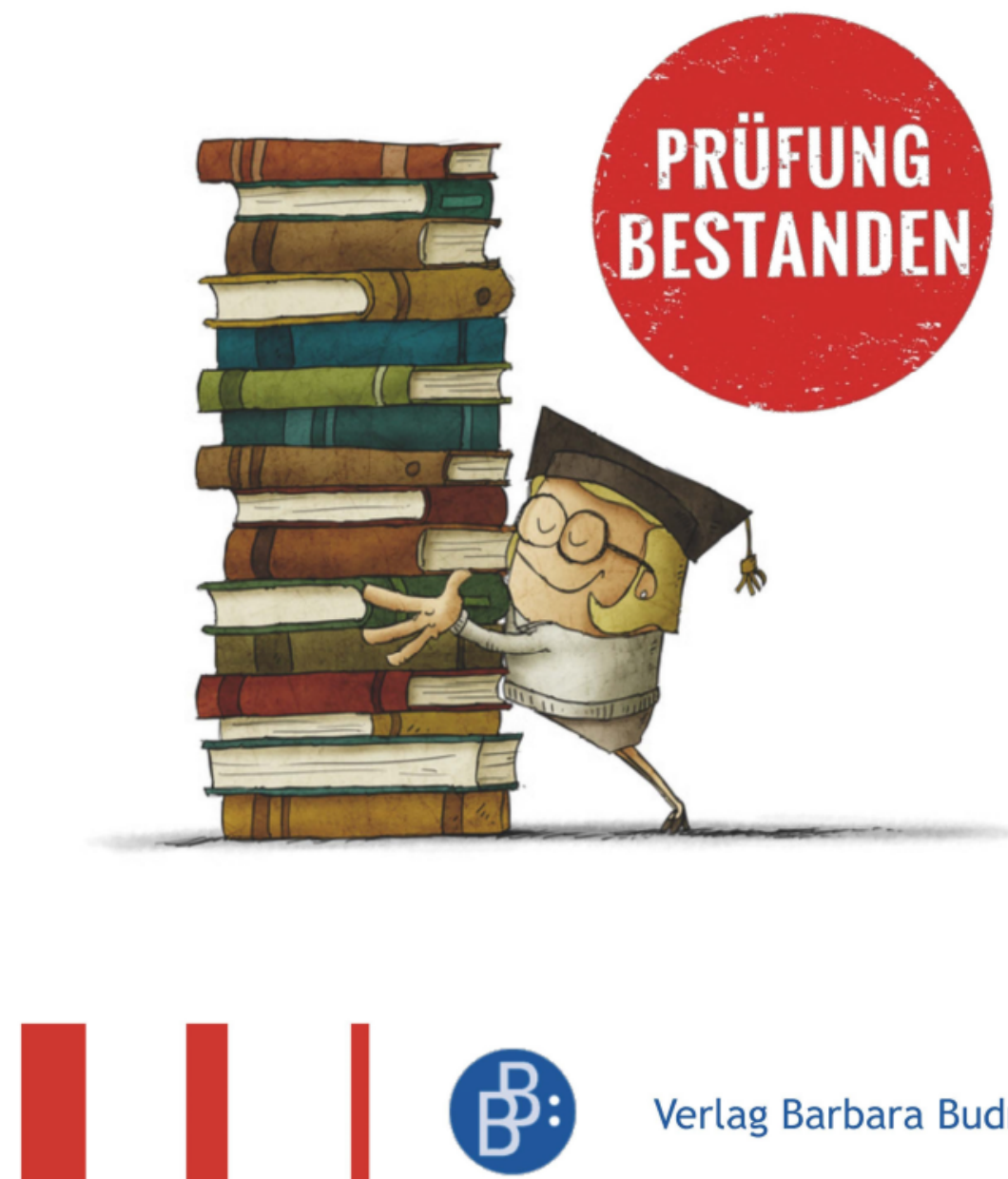

Verlag Barbara Budrich 
Inhaltsverzeichnis |

Werner Brandl

Editorial...... 2

Günther Koch

Wissenschaftliche Texte erschließen mit der Lesestrategie SQ3R 3

Martin Kornmeier

Kompetent wissenschaftlich schreiben

nach dem Gugelhupf-Prinzip.

Michael Booch

Zwischen Therapie und Beratung - Zur Rolle der Lehrkraft bei

der Präventions- und Interventionsarbeit von Essstörungen in der Schule.

Michaela Wölfer

Das Metabolische Syndrom im Jugendalter - Mögliche Ursachen

und Präventionsstrategien auf nationaler und internationaler Ebene

Julia Ernst

Aktuelle Herausforderungen bei der Schulverpflegung

an Gemeinschaftsschulen

Barbara Rieger

Essgenuss im Jugendalter.

Eva Steinfurth

Die Förderung des ethischen Ernährungsbewusstseins.

Andrea Balz \& Dina Limbach

Lernaufgaben und Unterrichtsmaterialien

zum neuen schweizerischen Lehrplan 21.

Birgit Happel

„Ich habe immer über meine Verhältnisse gelebt“ - Ein biografieanalytischer

Blick auf den Umgang mit Geld.

Tagungsankündigung

GFD Tagung 2017 Fachdidaktische Forschung zur Lehrerbildung 
Schulverpflegung an Gemeinschaftsschulen |

Julia Ernst

\section{Aktuelle Herausforderungen bei der Schulverpflegung an Gemeinschaftsschulen}

Dieser Artikel erläutert eine durchgeführte qualitative Einzelfallstudie, die sich mit aktuellen Herausforderung bei der Schulverpflegung an Gemeinschaftsschulen in Baden-Württemberg befasst. Insbesondere wird hierbei die Relevanz von ökologisch erzeugten Produkten genauer beleuchtet. Abschließend werden Hilfestellungen, welche den Einsatz von Bio-Lebensmitteln in der Schulverpflegung vorantreiben, aufgezeigt.

Schlüsselwörter: Schulverpflegung, Gemeinschaftsschulen, Bio-Produkte, Nachhaltigkeit

\section{Einleitung}

Seit dem Schuljahr 2012/13 ist im baden-württembergischen Schulsystem eine neue Schulform vorhanden - die Gemeinschaftsschule. Gemeinschaftsschulen sollen aufgrund ihrer ganztägigen Beschulung eine Schulverpflegung anbieten, dazu zählt vor allem eine warme Mittagsmahlzeit (vgl. Hahl, 2011).

Ergänzend zum Essensangebot zuhause kann der Nachwuchs ein ausgewogenes Mittagessen erhalten, das [...] einen hohen Bioanteil hat. Wer sich gut ernährt, lernt gut [...]. Und auch für die Zukunft der Kinder spielt das Verpflegungsangebot eine entscheidende Rolle. Denn gerade die ersten Lebensjahre sind wichtig für die Ausprägung eines gesunden, nachhaltigen Lebensstils. Schulen [...] tragen dabei eine wachsende Verantwortung. (Erhart et al., 2016, S. 4)

Folgt man dieser Argumentation, stellt der Einsatz von ökologisch erzeugten Lebensmitteln eine Herausforderung für Gemeinschaftsschulen dar. Eine qualitative Untersuchung soll eine Antwort darauf finden, welchen aktuellen Stellenwert ökologisch erzeugte Lebensmittel in der Schulverpflegung von Gemeinschaftsschulen besitzen.

\section{Im Kontext der Nachhaltigkeit}

Seit der internationalen Konferenz für Umwelt und Entwicklung 1992 in Rio de Janeiro ist die Begrifflichkeit der Nachhaltigkeit verbunden mit einem ökologischen, sozialen und ökonomischen Handeln (vgl. Erhart, 2009). 


\section{| Schulverpflegung an Gemeinschaftsschulen}

Die Vollwert-Ernährung von Koerber, Männle und Leitzmann (2012) greift diese drei Aspekte auf und fügt den Aspekt der Gesundheit ergänzend hinzu. Die Dimension Gesundheit miteinzubeziehen erachte ich im Hinblick auf ökologisch erzeugte Lebensmittel als sinnvoll und erwähnenswert. Methfessel sieht in dem viergliedrigen Konzept ${ }^{1}$ mit einer intra- und intergenerativen Orientierung die Möglichkeit einer gleichzeitigen Befolgung individueller und globaler Interessen (Methfessel, 2015). Verdeutlicht werden können diese komplexen Zusammenhänge und Wechselbeziehungen anhand der nachfolgenden Charakterisierung von nachhaltiger Ernährung:

Nachhaltige Ernährung ist ein offener, dynamischer und aktiv zu gestaltender Prozess [...] es handelt sich um ein historisch offenes gesellschaftliches Entwicklungsund Transformationskonzept. (Eberle et al., 2005, S. 11)

\subsection{Dimension Gesundheit}

Die aktuelle Entwicklung zur ganztägigen Beschulung stellt die Ernährung von Kindern und Jugendlichen aus Sicht der Schulen immer mehr in den Vordergrund und zugleich in deren Verantwortungsbereich. Folglich konkretisiert sich die Forderung nach einem gesundheitsförderlichen Verpflegungsangebot, welches die Schulen bereitstellen sollen (vgl. Schneider \& Metz, 2015). „Bio, vegetarisch, saisonal und regional sind genau die Komponenten, die es dazu braucht" (Erhart et al., 2016, S. 14). Allgemein soll damit sowohl die Förderung und Erhöhung des Wohlbefindens im alltäglichen Schulleben als auch die Erhaltung der schulischen Leistungsfähigkeit der Einzelnen erzielt werden. Auch eine Vorbeugung von Adipositas oder Karies sollte Ziel einer gesundheitsförderlichen Schulverpflegung sein. Näher betrachtet spielt auch eine bewusst erfahrbare Ernährungs- und Verbraucherbildung eine Rolle für die Angebotsgestaltung der Schulverpflegung. Denn nur, wenn die SchülerInnen einen Sinn hinter dem System erkennen, werden sie dieses erfolgreich tragen. Überdies dürfen die Lehrpersonen nicht in Vergessenheit geraten. Dementsprechend ist nicht nur eine Gesundheitsförderung bezogen auf die Heranwachsenden von besonderer Relevanz, sondern auch im Hinblick auf die Lehrerinnen und Lehrer, die gleichermaßen von der Ganztagsentwicklung betroffen sind (vgl. Schneider \& Metz, 2015; DGE, 2014).

\subsection{Dimension Wirtschaft}

In Bezug auf die Dimension Wirtschaft wird der Aspekt der Bezahlbarkeit in den Fokus genommen. Zum einen sollen aus finanzieller Sicht alle SchülerInnen am Essen teilnehmen können. Dadurch werden staatliche Fördermaßnahmen notwendig. Zum anderen ist für die Anbieter, wie den Caterern, die Wirtschaftlichkeit in Herstellung und Ausgabe der Speisen von hoher Bedeutung und steht im engen Zusammenhang mit den verkauften Portionen und der daraus resultierenden Rentabilität. Oft- 


\section{Schulverpflegung an Gemeinschaftsschulen |}

mals unvereinbar erscheinen dementsprechend gesundheitliche Aspekte mit den wirtschaftlichen, da mit meist gekauften Produkten - darunter fallen häufig Süßigkeiten - versucht wird das Verpflegungssystem in seiner Wirtschaftlichkeit zu stärken (Schneider \& Metz, 2015). Zudem ist für Großküchen der Energieverbrauch ein extremer Kostenfaktor. Ein geändertes Nutzungsverhalten trägt unter anderem dazu bei, dass sich die Energiebilanz in Großküchen wesentlich verbessert (vgl. Erhart et al., 2016). Hierbei wird die enge Verbindung zwischen den Dimensionen Wirtschaft und Umwelt deutlich.

Eine weitere wesentliche Thematik - nicht nur mit Blick auf die Kosten - ist das Vermeiden von Lebensmittelabfällen. Deshalb ist es für Großküchen essentiell, sich bei der Festlegung der Bestellmengen und Portionsgrößen nicht nur auf die Erfahrung zu verlassen, sondern eine detaillierte Kalkulation der Rezepte anzustellen. Folglich lassen sich sowohl die Einkaufs- und Entsorgungskosten senken als auch die zu entsorgenden Speise- und Lebensmittelreste reduzieren (vgl. Erhart et al., 2016).

\subsection{Dimension Gesellschaft}

Betrachtet man die gesellschaftliche Perspektive, so gewinnt die gemeinschaftliche Esssituation an Bedeutung: unterschiedliche Geschlechter, Kulturen, Religionen und verschiedene sozio-ökonomische Hintergründe sowie diverse Altersstufen treffen aufeinander bzw. vereinen sich zum gemeinsamen Essen.

Dahingehend muss auch die Schulverpflegung in ihrem Angebot gestaltet und auf die unterschiedlichsten Aspekte abgestimmt werden, um eine allgemeine Akzeptanz zu erreiche „Schulverpflegung, an der alle SchülerInnen selbstverständlich teilnehmen, kann $[\ldots]$ als wichtiger Beitrag zur Integration [...] angesehen werden“ (Schneider \& Metz, 2015, S. 119). Diese Dimension ist der Aspekt der sozialen Partizipation. Dementsprechend eröffnet sich die Möglichkeit, dass sich Eltern und SchülerInnen beteiligen können. Auch die Arbeitsbedingungen des Küchenpersonals sind nicht außer Acht zu lassen:

So kann sich jede Einrichtung fragen: Was kann ich besser machen? Wie sorge ich für ein angenehmes Arbeitsklima? Was kann ich tun, damit die Kinder das Essen in Gemeinschaft schätzen und davon profitieren? (Erhart et al., 2016, S. 14)

\subsection{Dimension Umwelt}

In allen gesellschaftlichen Bereichen sollen schädigende Einflüsse, die die Umwelt dauerhaft belasten, aufgedeckt und untersucht werden (vgl. Koerber et al., 2012).

Aspekte wie saisonale und regionale Lebensmittelerzeugung, der Anteil ökologisch erzeugter Lebensmittel, Verpackung, Lebensmittelabfälle sowie der Energieverbrauch bei Lagerung und Zubereitung beeinflussen die ökologische Bilanz des 


\section{| Schulverpflegung an Gemeinschaftsschulen}

Essens an der Schule. Insbesondere aus ökologischer Sicht wird für die Schulverpflegung unter anderem der Einsatz von ökologisch erzeugten Lebensmitteln empfohlen (vgl. Schneider \& Metz, 2015). Erhart et al. (2016) gehen noch weiter, indem sie behaupten, dass biologisch erzeugte Lebensmittel erst ihren Anteil für Mensch und Umwelt liefern, wenn sie aus der Region stammen.

In den einzelnen Dimensionen wurde die jeweilige Bedeutung einer nachhaltigen Schulverpflegung ersichtlich. Für die Kinder und Jugendliche, die den Großteil der Nutzer einer Schulverpflegung ausmachen, gilt weiterhin:

Ziel ist es, für die Heranwachsenden Essensituationen so zu gestalten, dass daraus ein Gemeinschaftserlebnis wird. Stein (2015) fordert ein ganzheitliches Ernährungsbewusstsein, welches sich auf gesunde Ernährung und nachhaltiges Handeln aller Beteiligten fokussiert. Dabei soll die Verpflegung zu einer ,,gelebten' Ernährungs- und Verbraucherbildung beitragen" (Schneider \& Metz, 2015, S. 118). Auf der Organisationsebene für die Schulmensa fordert Stein (2015) deshalb unter anderem ein Nahrungsmittelangebot aus zertifiziert ökologischer Landwirtschaft.

Ausgehend von der theoretischen Basis ist eine Schulverpflegung, bei der ökologisch erzeugte Lebensmittel zum Einsatz kommen, Bestandteil des Leitbildes einer nachhaltigen und gesunden Ernährung.

\section{Methodische Konzeption der Studie}

Als Forschungsmethode dienen hauptsächlich zwei qualitative Experteninterviews und ergänzend dazu eine teilnehmende Beobachtung ${ }^{2}$, damit die qualitative Einzelfallstudie mit Blick auf das Forschungsziel viele relevante Perspektiven, Dimensionen und Facetten erfassen kann (vgl. Lamnek, 2010). In besonderem Maße wird hierbei deutlich, dass eine ganzheitliche Erfassung von systemischen Zusammenhängen im Fokus steht.

Die ausgewählten Experten ${ }^{3}$ thematisieren die aktuellen Herausforderungen der Schulverpflegung und werden zugleich zu Akteuren, deren Aufgabe es ist, sich unter anderem mit dem Angebot einer Schulverpflegung auseinanderzusetzen. Bei der gewählten Art von Einzelfallstudie stehen Interaktionen eines Individuums zu anderen Individuen oder zu einer Ansammlung von Personen im Mittelpunkt (vgl. Lamnek, 2010). So spielen Erfahrungen der Befragten mit den jeweiligen Außenkontakten eine besondere Rolle.

Ziel der Forschung ist es, dass die Experten darüber berichten, inwiefern der Einsatz von ökologisch erzeugten Produkten gegenwärtig eine Rolle spielt. Die Experten können unterschiedlichen Tätigkeitsbereichen zugeteilt werden. Expertel (E1) ist eine stellvertretende Schulleitung einer Gemeinschaftsschule in der Rhein-NeckarRegion. An dieser Schule entstand auch die teilnehmende Beobachtung, welche jedoch im Vergleich mit den beiden Interviews für die Forschung nur eine untergeordnete Rolle spielt. Experte2 (E2) beliefert viele Kitas und Schulen mit einer warmen 


\section{Schulverpflegung an Gemeinschaftsschulen |}

Mittagsverpflegung. Er kann als ein typischer Vertreter unter den SchulbelieferungsUnternehmen angesehen werden, da er sich auf ein Verpflegungsangebot für Kinderund Jugendeinrichtungen spezialisiert hat.

Für diese Forschung wurden vorab Leitbilder formuliert, welche das Forschungsinteresse bzw. die Forschungsfrage abbilden sollen. Diese werden für den Leitfaden in Interviewfragen umformuliert.

Die erfassten Daten aus den Experteninterviews und der teilnehmenden Beobachtung werden mittels qualitativer Inhaltsanalyse ausgewertet, zum Beispiel mit Protokolltexten oder Transkriptionen. Bei der Anwendung der qualitativen Inhaltsanalyse werden den Texten Daten entnommen. Dadurch kommt es zu einer Extraktion von Rohdaten. Dementsprechend werden die Daten aufbereitet, sodass diese im Anschluss zur Analyse bereitstehen und eine Interpretation ermöglichen (vgl. Gläser \& Laudel, 2010).

Nach der Analyse und Diskussion der Ergebnisse werden in Anlehnung an die theoretische Basis der qualitativen Sozialforschung Hypothesen generiert.

\section{Diskussion des Forschungsergebnisses: Die Relevanz von ökologisch erzeugten Produkten in der Schulverpflegung}

In diesem Kapitel werden die Ergebnisse anhand von Leitbildern analysiert und interpretiert, diese wurden auf Grundlage des vorangegangenen Theorieteils entwickelt und sollen zur Beantwortung der Fragestellung beitragen. Dabei wird der Bezug zur Fachliteratur und anderen Studien hergestellt. Abschließend ergeben sich aus jedem Unterkapitel Hypothesen, welche im letzten Unterkapitel übersichtlich aufgelistet werden.

\subsection{Den Schülerlnnen sind ökologisch erzeugte Produkte besonders wichtig}

Dieser Abschnitt rückt die SchülerInnen als die Verbraucher und ihren Bezug zu ökologischen Erzeugnissen in den Fokus.

E2 stellt Vermutungen an, dass den SchülerInnen der Geschmack und eine warm gebliebene Speise auf dem Teller wichtiger sind als Bio-Produkte. Auch ein hoher Fleischgehalt spielt dabei eine Rolle. Zudem sind E2 keine Fälle bekannt, bei denen die SchülerInnen an der Essensausgabe nachfragen, was Bio bedeutet. Aber im gleichen Zuge merkt er die schwierige Überprüfbarkeit an, da die SchülerInnen heutzutage möglicherweise weniger Fragen stellen, sondern sich Hilfe im Internets suchen. Eine erkennbare Relevanz von Bio-Produkten bei SchülerInnen konnte durch die Experteninterviews ebenso wie bei der teilnehmenden Beobachtung nicht nachgewiesen werden. Für den Großteil der Schulverpflegungsnutzer stehen andere Aspekte 


\section{| Schulverpflegung an Gemeinschaftsschulen}

im Vordergrund ihres Interesses. Parallelen zu diesem Ergebnis werden auch bei der Sichtung der Fachliteratur ersichtlich. Es existieren beispielsweise Studien über die Relevanz des Geschmacks bei Kindern und Jugendlichen und über die Akzeptanz der Schulverpflegung (vgl. Lülfs-Baden \& Spiller, 2009). Aber keine Forschung nimmt Bezug zum Stellenwert von Bio-Produkten bei Kindern und Jugendlichen.

Hilfreich wären quantitative Erhebungen, die die Befragung von SchülerInnen oder auch Eltern vorsehen und deren Sichtweise repräsentativ darstellen.

\subsection{Beweggründe für den Einsatz von ökologisch erzeugten Lebensmitteln}

Betrachtet man die vier Interviewinhalte und die gesammelten Daten der teilnehmenden Beobachtung hinsichtlich der vier Dimensionen der Nachhaltigkeit, fällt auf, dass ökologisch erzeugte Lebensmittel vor allem mit der Dimension Gesundheit assoziiert werden, weil dahinter gesündere Lebensmittel vermutet werden. Der Blickwinkel auf die Umwelt wird in diesem Zusammenhang nicht berücksichtigt, sondern nur bei einer weiter gefassten Betrachtung auf die gesamte und allgemeinere Schulverpflegung, wie beispielsweise bei der Zubereitung der Speisen oder der Abfallproblematik (zusammengefasste Interviewinhalte von E1 und E2). Auch Hayn, Empacher und Halbes (2005) weisen in Anlehnung an Kuhnert et al. (2002) und Bruhn (2001) allgemeine Kaufmotive für ökologisch erzeugte Lebensmittel nach, die sich mit den Erkenntnissen aus dieser Studie decken: „Bei den Kaufmotiven haben Umweltschutzgründe deutlich an Bedeutung verloren, gesundheitliche Motive stehen klar an erster Stelle [...]" (Kuhnert et al., 2002; Bruhn, 2001 zit. nach Hayn et al., 2005, S. 16).

Aus dem Einsatz der ökologisch erzeugten Produkte werden vielmehr wirtschaftliche Vorteile gezogen. Hierbei sind die aus der Bio-Zulassung resultierenden Werbezwecke für ein Catering-Unternehmen zu nennen. Das Zeichen der BioZertifizierung ist deutlich sichtbar auf der Homepage (von E2) zu finden. Die unten aufgeführte Antwort von E2 hebt dies hervor:

[...] Wenn ich Bio-Catering und Kindergarten eingebe, konnte man uns vorher so gut wie nicht finden, weil vorher Bio einfach fehlte. Aber jetzt stehen wir unten den

Top fünf in Deutschland, rein von den Suchmaschinen und da geht es halt um ein Geschäft. (E2)

Entgegengesetzt dazu steht ein hoher finanzieller Aufwand, der mit der Einführung von Bio-Produkten in der Schulverpflegung auf Kommunen, Schulträger und Eltern zukommen kann. Im Zuge dessen thematisiert E1 Erschwernisse am Verhandlungstisch mit dem Schulträger bei der allgemeinen Planung und Auseinandersetzung mit der Thematik Schulverpflegung. Solche Verhandlungen fokussieren oftmals den finanziellen Aspekt, da die Finanzierung im Allgemeinen aus dem Eigenanteil des Endverbrauchers besteht (wird i. d. R. von den Eltern getragen) und einer Kosten- 


\section{Schulverpflegung an Gemeinschaftsschulen |}

übernahme durch den jeweiligen Schulträger (vgl. Jansen \& Schreiner-Koscielny, 2013). Diese Tatsache der Teilkostenübernahme durch den verantwortlichen Schulträger kann bereits ein Hindernis bei der Auswahl eines Caterings darstellen. Beispielsweise wird ein teures Unternehmen, welches hohe Kosten aufgrund des hohen Anteils an ökologisch erzeugten Lebensmitteln begründet, häufig im Vorfeld ausgeschlossen. Einen Kompromiss bilden für den Caterer einzelne Bio-Komponenten, da hierfür keine Preiserhöhung vollzogen werden muss. Nölting, Reimann und Strassner sprechen organisatorische Schwierigkeiten und die finanzielle Entscheidungseinschränkung in diesem Kontext zutreffend an:

Die Schulträger sind zuständig für die Organisation der Schulverpflegung als, äußere

Schulangelegenheit ${ }^{\prime}$. Sie sind in der Regel den Kommunen zugeordnet, die auch finanziell verantwortlich sind. Die unübersichtlichen Zuständigkeiten erschweren die

Einführung einer Schulverpflegung. (Nölting et al., 2010, S. 41)

Erhebungen in Österreich und Schweden (2005), die im Zuge der Ernährungswende die nachhaltige Ernährung in Schulen analysiert haben, kamen zu folgendem Ergebnis im Hinblick auf die Finanzierung von einem biologisch ausgerichteten Angebot: „[Die] Top-Down-Regelungen von Bundesländern oder Gemeinden [müssen] über einen gewissen Bio-Anteil [...] so ausgerichtet sein, dass die Kosten nicht steigen und dass die spezifischen Bedürfnisse der einzelnen Schulen Platz finden" (Mraz, Hofmann, Gruber \& Egger-Rollig, 2005, S. 57; Hervorhebung im Original).

Diese finanziellen Problematiken müssen also auf politischer Ebene behoben werden, um die Rahmenbedingungen für die „unmittelbaren“ Akteure rund um die Schule zu erleichtern.

Nicht außer Acht zu lassen sind mögliche finanzielle Problematiken beim Endverbraucher - in dem Fall bei den Eltern, die für die Verpflegung ihres Kindes aufkommen. E2 spricht von höheren Kosten, je größer der Bio-Anteil. Aufgrund der sozialen Ungleichheiten, die bereits das Schulleben der Kinder und Jugendlichen beeinflussen, können und wollen sich viele nicht das teuerste Verpflegungsangebot leisten. Obwohl jedem Sozialleistungsempfänger seit 2011 Beihilfen über das Bildungspaket zur Verfügung stehen (vgl. Jansen \& Schreiner-Koscielny, 2013), ist zu vermuten, dass Kommunen und Schulträger im Vorfeld des Engagements eines Catering-Unternehmens, auf den Endpreis für den Verbraucher achten, um den Einsatz solcher Zuschüsse einzugrenzen.

\subsection{Aktuelle Herausforderung der Schulverpflegung}

Aufgrund dessen, dass eine ganztägige Beschulung in Baden-Württemberg flächendeckend vorangetrieben wird, kommen zahlreiche neue Herausforderungen auf die Schulen zu, auch in Bezug auf die Schulverpflegung. So ist das Angebot der Mittagsverpflegung wenig strukturiert und das Fehlen von etablierten Programmen und Richtlinien erschwert die Lage (Clausen \& Kersting, 2010; Erhart et al., 2016). Die 


\section{| Schulverpflegung an Gemeinschaftsschulen}

Ansicht von E1 untermauert dieses Argument, da allein durch den geplanten Neubau noch zwei Jahre überwunden werden müssen und erst danach die räumlichen Kapazitäten und Strukturen dem Gesamtkonzept Ganztagsschule im vollen Maße entsprechen.

Im Hinblick auf die aktuellen Situationen in den Schulen und den biologisch erzeugten Lebensmitteln sehen Nölting et al. einen bedeutenden Zusammenhang: „Da sich Kompetenzen und Kapazitäten für die Schulverpflegung vielerorts noch im Aufbau befinden, ist der Einsatz ökologischer Produkte meist eine zusätzliche Herausforderung" (Nölting et al., 2010, S. 42). Setzt man dieses Zitat in Bezug auf beide Interviews, wird ersichtlich, weshalb ökologisch erzeugte Produkte nicht als gegenwärtige Chance oder Herausforderung angesehen werden: Zum einen, weil der Einsatz dieser Produkte nicht bewusst von der Schule erfolgt, womöglich gerade wegen der hohen Zahl an anderen zu meisternden Herausforderungen. Zum anderen fehlen oftmals ausgearbeitete Konzepte auf Länderebene oder kommunaler Ebene, die spezielle Kriterien und Richtlinien für die Schulverpflegung vorgeben, wodurch die gezielte Verwendung solcher Lebensmittel erleichtert werden könnte (Erhart et al., 2016; Jansen \& Schreiner-Koscielny, 2013).

Baden-Württemberg sieht keine gesetzlichen Vorschriften vor. In Teilen von Bayern beispielsweise sieht es etwas anders aus. Auch in Berlin existieren Kriterien, welche einen Bio-Anteil festsetzen (vgl. Nölting, Reimann \& Strassner, 2009; Seegers, 2007). Die Deutsche Gesellschaft für Ernährung (DGE) mit ihren formulierten Standards bietet eine der wenigen Alternativen zur Orientierung. Diese Standards werden jedoch nur empfehlend ausgesprochen und nicht verbindlich vorgeschrieben.

Bemerkenswert an dieser Stelle sind die Erfahrungen des Caterers (E2), dass die Schulen die Verantwortung eines Verpflegungsangebots häufig unzureichend tragen und dadurch auch kaum eine Kooperation zwischen dem Belieferungsdienstleister und der Schule stattfindet. Auch E1 sieht in der Kooperation lediglich das Abbestellen großer Essensmengen. Dies führt deutlich vor Augen, dass eine wirkliche Übernahme von Ernährungsverantwortung die Schulen noch zu selten beschäftigt. Der schnelle Wandel, der eine Enthäuslichung von Ernährung mit sich bringt und so die Ernährungsverantwortung aus dem privaten Bereich hinein in die Öffentlichkeit verlagert (vgl. Mraz et al., 2005), steht dem Verantwortungsübernahmeprozess der Schulen wahrscheinlich zusätzlich im Weg. Nichtsdestotrotz sehen Nölting et al. (2009) eine deutliche Tendenz zu einer professionellen Umsetzung, wenn CateringUnternehmen die Verantwortung und der Auftrag übergeben werden. Der Voraussetzungen, dass sich die Anbieter ihrer Verantwortung für eine Außer-HausVerpflegung mit einem gesunden wohlschmeckenden Angebot bewusst sind und einen hohen Standard der Qualität verfolgen (vgl. Schönberger, 2011), werden somit in den Augen von Nölting et al. erfüllt. 


\section{Schulverpflegung an Gemeinschaftsschulen |}

\subsection{Im Hinblick auf die Schulverpflegung erfolgt eine gezielte Auseinandersetzung mit der Thematik „ökologisch erzeugte Lebensmittel".}

Das Nichtwissen seitens des Interviewpartners E1 um die Verwendung von ökologisch erzeugten Produkten in der gelieferten Mittagsmahlzeit gilt als Indiz dafür, dass keine Auseinandersetzung auf Schulleitungsebene mit dieser Thematik erfolgt.

Auch eine bundesweite Erhebung bestätigt den oben genannten Aspekt: Die Auswertung der 3530 Fragebögen ergab, dass es allein 24 Prozent der Schulleitungen nicht bekannt ist, ob biologisch erzeugte Lebensmittel überhaupt bei der Essenszubereitung berücksichtigt werden (Arens-Azevedo, Schillmöller, Hesse, Paetzelt \& Roos-Bugiel, 2015).

Bei einer weiteren bundesweiten quantitativen Erhebung (2007/2008) wurden Befragungsbögen an 8733 Ganztagsschulen verschickt. 2920 rückläufige Fragebögen konnten zur Analyse herangezogen werden. Die Ergebnisse der Befragung werden in dem Beitrag von Arens-Azevedo im Jahrbuch Ganztagsschule 2011 dargestellt. 770 Schulen wollen keine Angaben zum Einsatz von Bio-Lebensmitteln machen und von den restlichen 2190 befragten Schulen geben 37,5 Prozent an, dass keine ökologisch erzeugten Lebensmittel zum Einsatz kommen (vgl. Arens-Azevedo, 2011)

Folglich ist die Tatsache, dass wenig Auseinandersetzung mit dem Einsatz von Bio-Produkten auf Schulleitungsebene erfolgt, auch deutschlandweit ein bemerkenswertes Phänomen.

E2 bemängelt in seinen Ausführungen, dass die Schulen sich keine eigenen Kriterien einer Schulverpflegung setzen. Zudem setzen sich die Schulen im Voraus einer geeigneten Verpflegungseinführung zu wenig damit auseinander, wobei E2 darin eine große Bedeutung für eine gelingende Schulverpflegung sieht. Denkbar sind hier definierte Kriterien, die sich explizit auf ökologisch erzeugte Lebensmittel beziehen. Erhart et al. teilen diese Ansicht:

Ein gut durchdachtes Konzept ist ausschlaggebend für den Erfolg. Deshalb sollte jede Einrichtung möglichst frühzeitig entscheiden, welche Ziele sie erreichen will. Sollen es 100 Prozent Biogerichte sein oder gerade beim Einstieg nur einzelne Komponenten in Bioqualität? Welche Ansatzpunkte gibt es für nachhaltiges Handeln in der Schule $[\ldots]$ ? Wofür steht der Ökolandbau [...] All diese Fragen gilt es bereits in der Planungsphase zu klären. (Erhart et al., 2016, S. 13)

Im Gespräch mit E2 wird auch die Elternsicht beleuchtet. Er zieht Vergleiche zu anderen Verpflegungseinrichtungen mit Kindern, wie Krippen und Kitas. Hierbei betont er immer wieder, dass aufgrund der hohen Nachfrage von Bio-Produkten die Einführung der Bio-Komponenten stattfand ${ }^{4}$. Jedoch sind deutliche Unterschiede zu verzeichnen und lassen sich folgendermaßen zusammenfassen: Je älter die Kinder werden, umso geringer ist die Nachfrage nach ökologisch erzeugten Lebensmitteln. 


\section{| Schulverpflegung an Gemeinschaftsschulen}

Als Gründe nennt E2 die geringer werdende aktive Mitgestaltung und Mitbestimmung seitens der Eltern, die mit der abnehmenden Anzahl an Elternabenden und Elternbeiratssitzungen im Schulalter der Kinder einhergeht.

\subsection{Die generierten Hypothesen der Ergebnisanalyse}

Als abschließendes Kapitel werden aus den vorangegangenen Abschnitten die Hypothesen übersichtlich aufgelistet. Diese Hypothesen haben den Anspruch in weiteren Forschungen überprüft zu werden, bei denen auch quantitative Vorgehensweisen denkbar sind.

- Für die SchülerInnen liegen ökologisch erzeugte Lebensmittel weniger im Interessenshorizont. Die Schule könnte dies verändern, indem mit einer bewussten Ernährungsbildung dieses Ziel verfolgt wird.

- Motive einer Verwendung von Bio-Produkten ergeben sich aus gesundheitsfördernden Betrachtungsweisen, wobei die günstigen Facetten der Umweltverträglichkeit außer Acht gelassen werden.

- Eine gute Wirtschafts- und Sozialverträglichkeit von Bio-Produkten wird kaum gesehen.

- Aktuelle Herausforderungen der Schulverpflegung an Gemeinschaftsschulen liegen nicht im Bereich einer Einführung bzw. Verwendung von ökologisch erzeugten Produkten. Sie konzentrieren sich vielmehr auf Rahmenbedingungen, dass überhaupt eine Schulverpflegung gewährleistet werden kann.

- Akteure, wie Schulträger, Schulleitung, SchülerInnen der Gemeinschaftsschulen und deren Eltern setzen sich nicht mit ökologischen Erzeugnissen als Alternative zu konventionellen Produkten im Kontext der Schulverpflegung auseinander.

Die Ergebnisse dieser qualitativen Forschung verdeutlichen, dass der anfänglichen Forschungsfrage, welche zunächst nur auf die ökologischen Produkte abzielte, nur gerecht werden konnte, indem ein offener und weiter Blickwinkel gewahrt wurde und somit der gesamte Bereich der Schulverpflegung nie in Vergessenheit geriet. Nur so konnten die Komplexität und die vielen verschiedenen Herausforderungen der Schulverpflegung zum Ausdruck kommen.

Hinsichtlich des aktuellen Stellenwerts von ökologischen Erzeugnissen in der Schulverpflegung, gibt es Musterbeispiele. Es handelt sich hierbei um forcierende Initiativen und Beispielschulen, die das vorliegende ernüchternde Ergebnis nicht widerspiegeln. Ein solches Vorbild, das mit dem Einsatz ökologisch erzeugter Lebensmittel bewusst im Sinne der Nachhaltigkeit handelt bzw. das nachhaltige Handeln in Schulverpflegungssituationen initiativ unterstützt, soll im Folgenden erläutert werden. 


\section{Schulverpflegung an Gemeinschaftsschulen |}

\section{Ausblick: „Bio kann jeder!“}

Der Ausbau der Ganztagsbetreuung birgt für die Schulen und Kitas die große Chance, ihre Speiseangebote kindgerecht zu gestalten und dabei von Beginn an auf Bio zu setzen. Das ist machbar und verursacht kaum Mehrkosten - vorausgesetzt man weiß, wie es funktioniert. (Erhart et al., 2016, S. 4)

Bio kann jeder! ist ein Projekt auf bundesweiter Ebene und ist ausgezeichnet als offizielle Maßnahme der UN-Weltdekade Bildung für nachhaltige Entwicklung (Erhart, 2009). Initiiert wurde es im Kontext vom Bundesprogramm Ökologischer Landbau und anderer Formen nachhaltiger Landwirtschaft (BÖLN). Es stellt die erste Unterstützung auf Seiten der bundesweiten politischen Ebene dar (vgl. Nölting et al., 2009).

Hauptziele der Kampagne sind die „Stärkung und Ausdehnung der ökologischen und nachhaltigen Land- und Lebensmittelwirtschaft in Deutschland" (Erhart et al., 2016, S. 5).

Im ganzen Land werden seit dem Projektstart im Jahre 2004 Workshops für Vertreter und Interessierte aus Schulen und anderen Betreuungseinrichtungen für Kinder und Jugendliche angeboten. Ebenfalls soll das erweiterte Umfeld - beispielsweise für Trägerschaften oder Caterer - angesprochen werden. Diese Workshops werden nach Länderebene strukturiert und organisiert, um den Teilnehmern die Handlungsmöglichkeiten in der Region aufzuzeigen.

Als Basiselemente der Workshops dienen ein funktionierender Informations- und Erfahrungsaustausch im Umgang mit biologisch erzeugten Lebensmitteln in der Außer-Haus-Verpflegung für Kinder und Jugendliche (vgl. Erhart, 2009; Erhart et al., 2016). Des Weiteren wird der Aufbau von Netzwerken der einzelnen Akteure rund um die Schulverpflegung angestrebt, um regionale Zusammenarbeit zu forcieren. Dadurch werden auf drei Ebenen der Nachhaltigkeit - der ökonomischen, der ökologischen und der sozialen - die Voraussetzungen begünstigt, indem geeignete Kooperations- und Vertragspartner gesucht werden. Zusätzlich wird in den Workshops das theoretische Wissen in einem praktischen Teil vertieft, in dem Besichtigungen von Schulen und Bauernhöfen mit ökologischer Orientierung sowie Übungen in der Nahrungszubereitung vorgesehen sind. Das präsentierte Verpflegungsangebot der einzelnen Praxisbeispiele und somit auch der Anteil der Bio-Lebensmittel sind vielfältig. Damit wird ein breites Spektrum an Umsetzungsmöglichkeiten veranschaulicht und auf den regionalen Bedarf angepasst. So können zahlreiche Anregungen in den eigenen Verpflegungsalltag mitgenommen werden (vgl. Erhart, 2009; Erhart et al., 2016).

Auf der Internetseite werden mehrere Schulen als Praxisbeispiele vorgestellt. Diese Beispiele machen deutlich, dass eine Auseinandersetzung mit dem Thema „nachhaltige Ernährung und ökologisch erzeugte Produkte“ erfolgsversprechend und zielführend sein kann. 


\section{| Schulverpflegung an Gemeinschaftsschulen}

\section{Fazit}

Ausgehend von der theoretischen Basis ist eine Schulverpflegung, bei der ökologisch erzeugte Lebensmittel zum Einsatz kommen, Bestandteil der Idealvorstellung einer nachhaltigen Ernährung. Dabei stellt eine nachhaltige Ernährung eine wichtige Voraussetzung für eine gesunde Entwicklung der Heranwachsenden dar, sie fördert das Umweltbewusstsein gleichermaßen wie die soziale Verträglichkeit und achtet auf das ökonomische Handeln der Akteure in der Schulverpflegung. Zudem könnte sich das Bio-Marktpotenzial mit dem Bereich Schulverpflegung bedeutend vergrößern (Nölting et al., 2010).

Die Realität, die durch die qualitative Studie aufgedeckt wird, ist eine andere: Ein geringer Stellenwert der ökologisch erzeugten Produkte ist anhand der ausgewählten Fallbeispiele erkennbar. Die Anlässe der einzelnen Akteure, BioLebensmittel in ihr Verpflegungsangebot aufzunehmen, werden weniger von Ansprüchen eines Nachhaltigkeitsbewusstseins geleitet. Die untersuchten Einzelfälle lassen vermuten, dass es in der ganzen baden-württembergischen Gemeinschaftsschul-Landschaft ähnlich aussieht, da die jüngste Umstrukturierung des Schulsystems in jeder Hinsicht eine Vielzahl an Herausforderungen aufgeworfen hat. Die Gemeinschaftsschulen in Baden-Württemberg müssen mehr in den Fokus genommen werden, da die nachhaltige Ernährung in der Schulverpflegung eine noch sehr untergeordnete Rolle spielt. Quantitative Erhebungen müssten die schlussgefolgerten Hypothesen dieser qualitativen Studie überprüfen, um einen repräsentativen Anspruch zu erreichen.

Die Ergebnisse machen deutlich, dass aufgrund der großen Anzahl an Herausforderungen für die Schulverpflegung an Gemeinschaftsschulen nur ein kleiner exemplarischer Einblick in diese Thematik erfolgen konnte.

Wird die Aufmerksamkeit mehr in Richtung einer nachhaltigen Schulverpflegung gelenkt und findet eine Anpassung des Angebots statt, so tritt in näherer $\mathrm{Zu}-$ kunft möglicherweise auch die Wertschätzung der Mensanutzer bezüglich eines guten Essens ein (Heindl, 2008). Impulsgeber für eine dynamische, hochwertige und nachhaltige Schulverpflegung können ein Umdenken auf politischer Ebene sowie das Gründen bzw. die Inanspruchnahme von Initiativen und Projekten sein. Ziel dieser Maßnahmen sollte sein, dass eine Verknüpfung der einzelnen Akteure, die sich aus unterschiedlichen Perspektiven mit der Schulverpflegung befassen, erfolgt. Eine große Bedeutung kommt den regionalen landwirtschaftlichen Gegebenheiten zu, da die Kooperation mit Bio-Bauern aus der Umgebung ökonomische, ökologische und soziale Aspekte der Nachhaltigkeit positiv begünstigen.

Trotz der Komplexität und der täglichen Herausforderungen, liegt in der Schulverpflegung das Potential, Ernährungsthemen in das Bewusstsein aller Akteure zu rücken und nicht zuletzt den SchülerInnen etwas Gutes zu tun. Hierfür soll ökologisch erzeugten Produkten eine besondere Aufmerksamkeit geschenkt werden, da 


\section{Schulverpflegung an Gemeinschaftsschulen |}

flexible Umsetzungsmöglichkeiten und Unterstützungsangebote bereits vorhanden sind und auf ihren verstärkten Einsatz warten.

\section{Anmerkungen}

1 Koerber hat eine fünfte Dimension, die Kultur, der nachhaltigen Ernährung hinzugefügt (vgl. Koerber, 2014). Diese wird in diesem Artikel nicht als eigenständige Dimension aufgenommen.

2 Die teilnehmende Beobachtung spielt eine geringere Rolle als die leitfadengestützten Experteninterviews. Sie dient lediglich der Sammlung von Informationen über Prozesse, Organisationstrukturen, Handlungsabläufe und Interaktionsmuster mit Blick auf die Schulverpflegung, um „strukturelle Zusammenhänge“ (Legewie, 1995, S.191) identifizieren zu können.

3 Mit Experten sind Fachfrauen und Fachmänner gemeint, die aufgrund ihrer unmittelbaren beruflichen Beteiligung über entsprechend professionelle Expertise verfügen. Deshalb entsteht ,aufgrund seiner [des Experten] individuellen Position und seiner persönlichen Beobachtungen eine besondere Perspektive auf den jeweiligen Sachverhalt" (Gläser \& Laudel, 2010, S. 11).

4 E2 führte zu der Thematik eine Online-Umfrage unter den Eltern durch.

\section{Literatur}

Arens-Azvedo, U. (2011). Verpflegung an deutschen Ganztagsschulen. Organisation und Strukturen. In S. Appel (Hrsg.), Mehr als Schule oder doch: mehr als Schule? Jahrbuch Ganztagsschule, 2011. (S. 127-139). Schwalbach am Taunus: Wochenschau.

Arens-Azvedo, U., Schillmöller, Z., Hesse, I., Paetzelt, G. \& Roos-Bugiel, J. (2015). Qualität der Schulverpflegung. Bundesweite Erhebung. Abschlussbericht. Unter Mitarbeit von M. Glashoff. Hg. v. Bundesministerium für Landwirtschaft und Ernährung. Hamburg.

https://www.in-form.de/fileadmin/redaktion/Publikationen/ pdfs/20150625INFORM_StudieQualitaetSchulverpflegung.pdf

Clausen, K. \& Kersting, M. (2010). Empfehlungen für die Mittagsverpflegung in der Ganztagsschule.

http://www.familienhandbuch.de/gesundheit/ernaehrungkindheit/empfehlungenfuerdiemittagsverpflegung.php

Deutsche Gesellschaft für Ernährung e.V. (DGE) (2014). DGE-Qualitätsstandard für die Schulverpflegung (4.Aufl.). Bonn.

http://www.schuleplusessen.de/service/medien.html?eID=dam_frontend_push\&d ocID $=1046$

Eberle, U., Fritsche, U., Hayn, D., Rehaag, R., Simshäuser, U., Stieß, I. \& Waskow, F. (2005). Nachhaltige Ernährung. Ziele, Problemlagen und Handlungsbedarf im 


\section{| Schulverpflegung an Gemeinschaftsschulen}

gesellschaftlichen Handlungsfeld Umwelt-Ernährung-Gesundheit. Diskussionspapier Nr.4. In Öko-Institut (Hrsg.). Ernährungswende.

http://www.isoe.de/ftp/EW_DP_Nr4.pdf

Erhart, A. (2009). Nachhaltigkeit in der Schulverpflegung. Ernährung im Fokus, 09 (4), 138-142.

Erhart, A., Lange-Fricke, I., Weiler, N. \& Zurek, C. (2016). Bio-Verpflegung in Kindertagesstätten und Schulen. Hrsg. v. Bundesanstalt für Landwirtschaft und Ernährung und Bundesprogramm Ökologischer Landbau und andere Formen nachhaltiger Landwirtschaft (BÖLN). Bonn.

Gläser, J. \& Laudel, G. (2010). Experteninterviews und qualitative Inhaltsanalyse. Als Instrumente rekonstruierender Untersuchungen (4. Aufl.). Wiesbaden: Springer VS. https://doi.org/10.1007/978-3-531-91538-8

Hahl, M. (2011). Ganztagsschulentwicklung in Baden-Württemberg. In S. Appel (Hrsg.), Mehr als Schule oder doch: mehr als Schule? Jahrbuch Ganztagsschule, 2011 (S. 152-159). Schwalbach am Taunus: Wochenschau.

Hayn, D., Empacher, C. \& Halbes, S. (2005). Trends und Entwicklungen von Ernährung im Alltag. Ergebnisse einer Literaturrecherche. Materialband Nr.2. Unter Mitarbeit von Annett Höpfner und Gudrun Seltmann. In Öko-Institut (Hrsg.), Ernährungswende. http://www.ernaehrungswende.de/pdf/ernwend_matband_2.pdf

Heindl, I. (2008). Kulinaristik und Allgemeinbildung. In A. Wierlacher (Hrsg.), Kulinaristik. Forschung - Lehre-Praxis (S. 129-146). Berlin: Lit.

Jansen, C. \& Schreiner-Koscielny, J. (2013). Schulverpflegung in Deutschland. aktueller Stand, Vorgaben und Entwicklungen. Ernährungs-Umschau, 13 (3), M158M164.

Koerber, K. v. (2014). Fünf Dimensionen der nachhaltigen Ernährung und weiterentwickelte Grundsätze. Ein Update. Ernährung im Fokus, 14 (09-10), 260-266. https://www.aid.de/_data/files/eif_2014_09-10_5-dimensionen_nachhaltigeernaehrung.pdf

Koerber, K. v., Männle, T. \& Leitzmann, C. (2012). Vollwert-Ernährung. Konzeption einer zeitgemäßen und nachhaltigen Ernährung (11. Aufl.). Stuttgart: Haug.

Lamnek, S. (2010). Qualitative Sozialforschung. Lehrbuch (5., überarb. Aufl.). Weinheim: Beltz.

Legewie, H. (1995). Feldforschung und teilnehmende Beobachtung. In U. Flick (Hrsg.), Handbuch qualitative Sozialforschung. Grundlagen, Konzepte, Methoden und Anwendungen. (S. 189-193, 3., neu ausg. Aufl.). Weinheim: Beltz PVU.

Lülfs-Baden, F. \& Spiller, A. (2009). Warum die Schüler nicht in die Mensa gehen. Zur Akzeptanz der Schulverpflegung. Ernährungs-Umschau, 09 (9), 506-513.

Methfessel, B. (2015). Welche Moral hätten Sie denn gerne? Essen im Konflikt zwischen unterschiedlichen Anforderungen an die Lebensführung. In G. Hirschfel- 


\section{Schulverpflegung an Gemeinschaftsschulen |}

der (Hrsg.), Was der Mensch essen darf. Ökonomischer Zwang, ökologisches Gewissen und globale Konflikte (S. 83-100). Wiesbaden: Springer VS.

https://doi.org/10.1007/978-3-658-01465-0_6

Mraz, G., Hofmann, R., Gruber, M. \& Egger-Rollig, E. (2005). Nachhaltige Ernährung in Schulen. Fallbeispiele aus Österreich und Schweden. Materialband Nr.5. In Öko-Institut (Hrsg.), Ernährungswende.

http://www.ernaehrungswende.de/fr_ver.html

Nölting, B., Reimann, S. \& Strassner, $\bar{C}$. (2009). Bio-Schulverpflegung in Deutschland. Ein erster Überblick. Unter Mitarbeit von M. Lukas. Zentrum Technik und Gesellschaft. Berlin (Diskussionspapier 30/09). https://www.tu-berlin.de/uploads/media/Nr_30_Noelting.pdf

Nölting, B., Reimann, S. \& Strassner, C. (2010). Mit Bio Schule machen. AußerHaus-Verpflegung. In Stiftung Ökologie und Landbau (Hrsg.), Ökologie und Landbau (S. 41-43). Bd.154, 2. München: oekom.

http://orgprints.org/17145/1/Noelting_Reimann_Strassner_2010_1003_\%C3\%96 L_Bioschulverpflegung final_\%282\%29.pdf

Schneider, K. \& Metz, M. (2015). Essen in der Schule. nachhaltige Schulverpflegung und Partizipation. In J. Schockemöhle \& M. Stein (Hrsg.), Nachhaltige Ernährung lernen in verschiedenen Ernährungssituationen. Handlungsmöglichkeiten in pädagogischen und sozialpädagogischen Einrichtungen (S. 117-127). Bad Heilbrunn: Klinkhardt.

Schönberger, G. (2011). Mahlzeiten neu denken. In G. Schönberger \& B. Methfessel (Hrsg.). Mahlzeiten. Alte Last oder neue Lust? (S. 39-52). Wiesbaden: Springer VS. https://doi.org/10.1007/978-3-531-92886-9

Seegers, I. (2007). Tafel-Freuden? Das Essen an deutschen Schulen. Dossier zur Situation der Schulverpflegung in Deutschland. Hrsg. v. Verbraucherzentrale Bundesverband e.V. Fachbereich Gesundheit und Ernährung. Berlin.

Stein, M. (2015). Essenssituationen in der Schule gestalten als Teil einer wertebasierten Schulentwicklung. In J. Schockemöhle \& M. Stein (Hrsg.), Nachhaltige Ernährung lernen in verschiedenen Ernährungssituationen. Handlungsmöglichkeiten in pädagogischen und sozialpädagogischen Einrichtungen (S.105-116). Bad Heilbrunn: Klinkhardt.

\section{Verfasserin}

Julia Ernst

Lehramtsanwärterin

E-Mail: jernst-k17s@seminar-mannheim.de 